\title{
Dual HER2 blockade in the neoadjuvant and adjuvant treatment of HER2-positive breast cancer
}

\author{
This article was published in the following Dove Press journal: \\ Breast Cancer: Targets and Therapy \\ 22 September 2015 \\ Number of times this article has been viewed
}

\author{
Pooja Advani' \\ Lauren Cornell ${ }^{2}$ \\ Saranya Chumsri ${ }^{1}$ \\ Alvaro Moreno-Aspitia' \\ 'Division of Hematology and \\ Oncology, ${ }^{2}$ Department of Internal \\ Medicine, Mayo Clinic, Jacksonville. \\ FL, USA
}

Correspondence: Alvaro Moreno-Aspitia Department of Hematology and

Oncology, Mayo Clinic, 4500 San Pablo

Road, Jacksonville, FL 32224, USA

Tel + I 904953729 |

Fax + I $90495323 \mid 5$

Email morenoaspitia.alvaro@mayo.edu

\begin{abstract}
Human epidermal growth factor receptor 2 (HER2) is a tyrosine kinase transmembrane receptor that is overexpressed on the surface of $15 \%-20 \%$ of breast tumors and has been associated with poor prognosis. Consistently improved pathologic response and survival rates have been demonstrated with use of trastuzumab in combination with standard chemotherapy in both early and advanced breast cancer. However, resistance to trastuzumab may pose a major problem in the effective treatment of HER2-positive breast cancer. Dual HER2 blockade, using agents that work in a complimentary fashion to trastuzumab, has more recently been explored to evade resistance in both the preoperative (neoadjuvant) and adjuvant settings. Increased effectiveness of dual anti-HER 2 agents over single blockade has been recently reported in clinical studies. Pertuzumab in combination with trastuzumab and taxane is currently approved in the metastatic and neoadjuvant treatment of HER2-positive breast cancer. Various biomarkers have also been investigated to identify subsets of patients with HER2-positive tumors who would likely respond best to these targeted therapy combinations. In this article, available trial data regarding efficacy and toxicity of treatment with combination HER2 agents in the neoadjuvant and adjuvant setting have been reviewed, and relevant correlative biomarker data from these trials have been discussed.
\end{abstract}

Keywords: HER2, dual blockade, neoadjuvant, adjuvant, breast cancer, trastuzumab

\section{Introduction}

Human epidermal growth factor receptor 2 (HER2) is a tyrosine kinase transmembrane receptor that is overexpressed on the surface of $15 \%-20 \%$ of breast tumors and plays a key role in intracellular signaling pathways of tumor cells. ${ }^{1}$ HER2 expression has been associated with aggressive disease and worse prognosis. ${ }^{2}$ However, HER2 does not function independently; instead, it is part of a family of tyrosine kinase receptors that also includes HER1 (epidermal growth factor receptor [EGFR]), HER3, and HER4. These receptors homo/heterodimerize with one another, thereby allowing auto-phosphorylation of tyrosine molecules, which then propagate intracellular signaling via the mitogen-activated protein kinases (HER1/HER2 heterodimers) and phosphoinositide 3-kinase (PI3K) pathways (HER2/HER3 heterodimers). HER2 is unique in that it is able to dimerize with any of the other three receptors and does not require ligand binding for activation. ${ }^{3}$ On the other hand, HER3 has weak intrinsic tyrosine kinase activity, but forms heterodimers with HER2, leading to the strongest activation signal. ${ }^{4}$

Trastuzumab (Herceptin ${ }^{\circledR}$; Genentech, Inc, South San Francisco, CA, USA) is a recombinant monoclonal antibody that binds to the extracellular domain IV of HER2, thereby inhibiting ligand-independent HER2 and HER3 signaling. It also activates antibody-dependent cellular toxicity (ADCC). ${ }^{5,6}$ The approval of trastuzumab in 1998 
has been a milestone in the treatment of HER2-positive breast cancer (BC). It has been shown to consistently improve disease-free survival (DFS) and overall survival (OS) in both early and metastatic BC (MBC). ${ }^{7-10}$ However, despite appropriate treatment with trastuzumab, up to $40 \%$ of patients with HER2 overexpression may be resistant to therapy (de novo and acquired resistance). ${ }^{11}$ Mechanisms for resistance to therapy are expansive and not completely understood. Suggested mechanisms include alterations in the presence of tyrosine kinase receptors, upregulation of downstream effectors, and/or activation of alternative pathways to bypass the HER2 inhibition. ${ }^{11}$

To combat these resistance pathways, several strategies have been employed, such as combination of anti-HER2 agents with chemotherapy, combination of two anti-HER2 agents with complimentary mechanisms of action, targeting HER2/HER3 dimerization, and testing of irreversible dual HER1/2 inhibitors, such as afatinib and neratinib. ${ }^{11}$ For example, pertuzumab (Perjeta ${ }^{\circledR}$; Genentech) binds in subdomain II of HER 2 to prevent dimerization between HER2 and HER3 and is currently US Food and Drug Administration (FDA) approved as a first-line therapy in combination with trastuzumab and chemotherapy in HER2-positive MBC and in the neoadjuvant setting. Lapatinib (Tykerb; GlaxoSmithKline plc, London, UK), another approved agent, is an oral small molecule dual inhibitor of tyrosine kinase activity of EGFR and HER2 that reversibly binds in the HER2 and/or HER1 intracellular domain. Additionally, lapatinib leads to HER2 accumulation at the cell surface, augmenting trastuzumab-dependent ADCC. ${ }^{12}$ But again, resistance to these drugs may develop within months of beginning treatment. ${ }^{13}$ Lapatinib is currently approved in women with HER2+ MBC that has progressed after trastuzumab treatment. ${ }^{14}$ Trastuzumab emtansine (T-DM1, Kadcyla $^{\circledR}$, Genentech) is a drug-antibody conjugate of trastuzumab and a cytotoxic moiety (DM1 or mertansine, a microtubule inhibitory agent), which is internalized after binding to HER2. Proteolytic degradation enables release of DM1, specifically in the lysosome of HER2overexpressing cells, causing cell death and thus reducing toxicity to unaffected tissues. ${ }^{15}$ Based on the phase III study of Trastuzumab Emtansine (TDM-1) versus Capecitabine Plus Lapatinib in Patients with HER2-Positive Locally Advanced or Metastatic Breast Cancer (EMILIA) trial results, TDM1 is currently approved for advanced/MBC in patients previously treated with trastuzumab and taxane therapy. ${ }^{16}$ Novel agents such as afatinib and neratinib (oral irreversible pan-HER inhibitor) are also currently being investigated. ${ }^{17}$
Another strategy to address drug resistance is to combine agents that target the HER family and work in a complementary fashion. Several preclinical studies have demonstrated synergistic activity of combinations of anti-HER agents, which may also help to overcome trastuzumab resistance. ${ }^{18-21}$ Arpino et $\mathrm{al}^{22}$ has demonstrated in animal models that the three-drug combination of gefitinib, pertuzumab, and trastuzumab is much more effective at eradicating HER2-overexpressing xenografts in mice than any of the single agents alone. Together, this combination blocks signaling from all HER receptor dimerization pairs, supporting the theory that combination HER-targeted therapy may be more successful than targeting only a single receptor domain. This was further evidenced in a study by Rimawi et $\mathrm{al}^{23}$ that showed that the combination of lapatinib and trastuzumab was also more effective in eradicating HER2-overexpressing xenografts. This preclinical evidence has led to the development of clinical trials to further evaluate the efficacy of dual HER2 blockade.

In a phase III, randomized study conducted by Blackwell et al, ${ }^{24}$ the combination of trastuzumab and lapatinib improved the progression-free survival (PFS) and clinical benefit rate when compared to lapatinib alone in patients with progression of $\mathrm{MBC}$ on prior trastuzumab-containing therapy. Clinical Evaluation of Pertuzumab and Trastuzumab (CLEOPATRA) trial demonstrated a significant improvement in OS to 56.5 months in the pertuzumab, trastuzumab, and docetaxel group versus 40.8 months in the trastuzumab and docetaxel group. Additionally, the PFS and duration of response were prolonged in the pertuzumab group, supporting the use of dual HER2 blockade in $\mathrm{MBC} .{ }^{25}$

Similar DFS and OS outcomes have been reported with preoperative (neoadjuvant) treatment of $\mathrm{BC}$ as administration of postoperative (adjuvant) systemic therapy. ${ }^{26,27}$ Additionally, neoadjuvant chemotherapy increases the rate of breast conservation surgery (BCS) and facilitates monitoring of responses to therapy, serving to demonstrate drug efficacy in a relatively short period of time. In randomized neoadjuvant studies, trastuzumab and chemotherapy-containing regimens have led to improved pathological complete response (pCR) at the time of surgery, which seems to correlate with improved disease outcomes, suggesting that pCR may serve as a surrogate marker of clinical benefit. ${ }^{28,29}$ Dual HER2 blockade has been explored in preoperative, metastatic, and adjuvant settings. In the current article, the neoadjuvant and adjuvant trials utilizing dual HER2 therapy, including relevant correlative 


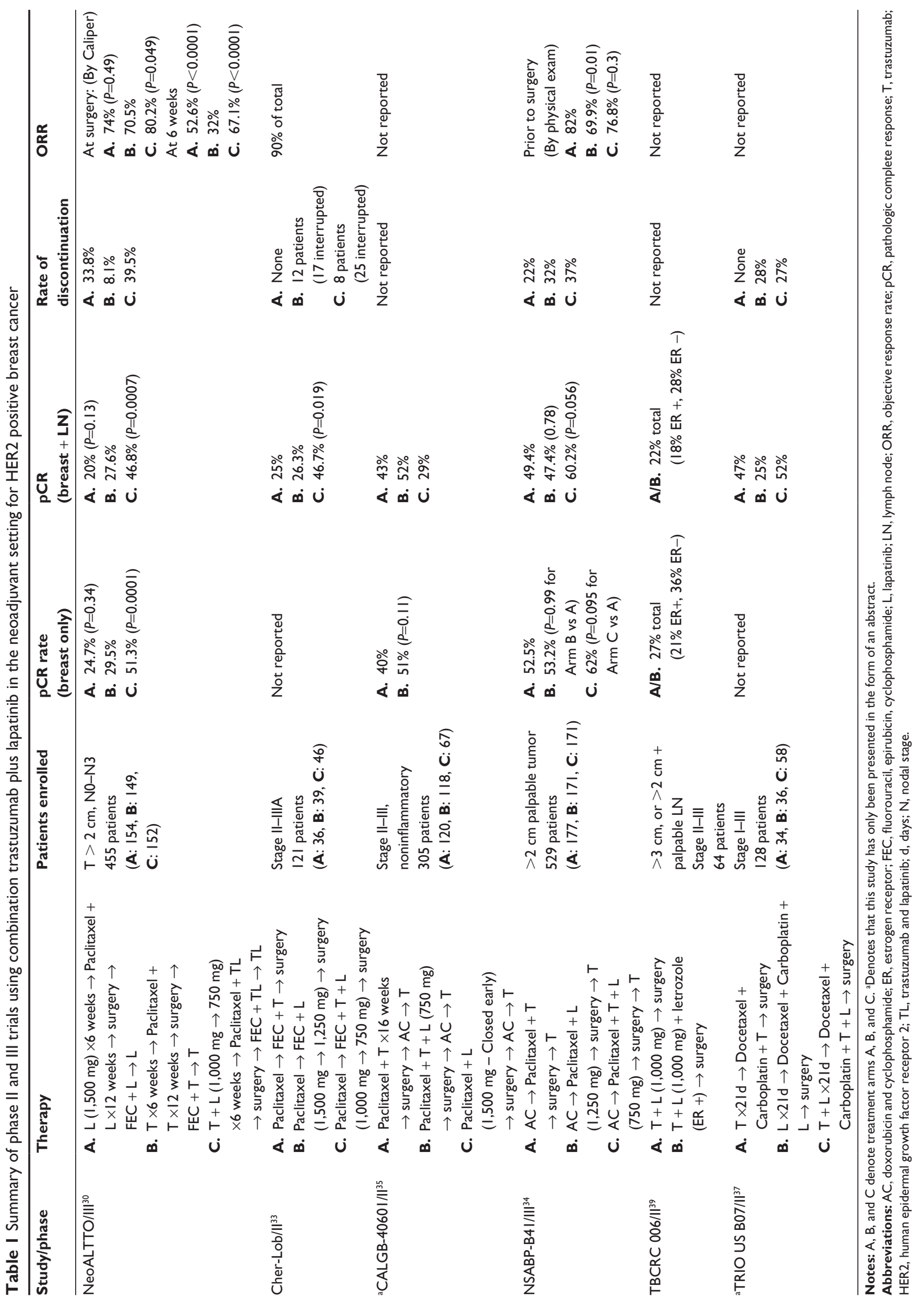


biomarker data, where available, have been reviewed in the following sections.

\section{Neoadjuvant trials Trastuzumab plus lapatinib Efficacy}

The combination of trastuzumab and lapatinib in the neoadjuvant setting has been studied by multiple trials with varying regimens of chemotherapy (Table 1). In the Neoadjuvant Lapatinib and/or Trastuzumab Treatment Optimisation (NeoALTTO) trial, a phase III, multicenter study, 455 women with operable HER $2+$ tumors measuring $>2 \mathrm{~cm}$ were randomized to receive either lapatinib, trastuzumab, or the combination for 6 weeks, followed by 12 weeks of paclitaxel in addition to the assigned HER2 therapy prior to scheduled breast surgery. ${ }^{30} \mathrm{After}$ surgery, all patients received three cycles of 5-fluorouracil, epirubicin, and cyclophosphamide (FEC), followed by the continuation of the initially assigned anti-HER2 therapy for an additional 34 weeks. The study's primary endpoint of pCR, defined as absence of invasive cancer in the breast at the time of surgery, was significantly higher in patients treated with lapatinib and trastuzumab than with trastuzumab alone ( $51.3 \%$ vs $29.5 \%$; $P=0.001$ ), supporting dual blockage of the HER pathway in the neoadjuvant setting. The odds ratio (OR) for $\mathrm{pCR}$ in the combination group relative to the trastuzumab-alone group was $2.6(P=0.0001)$. Also, no significant difference in pCR was seen between the lapatinib and trastuzumabalone groups ( $29.5 \%$ vs $24.7 \%)$. An improvement was seen in pCR rate in breast and axilla in the dual therapy group compared to trastuzumab alone ( $46.8 \%$ vs $27.6 \%$; OR 2.39 , $P=0.0007)$ and improved objective response rate (complete response $[\mathrm{CR}]+$ partial response $[\mathrm{PR}])$ at 6 weeks for both arms containing lapatinib compared to trastuzumab $(52.6 \%$ lapatinib alone, $67.1 \%$ combination, and $30.2 \%$ trastuzumab alone; $P<0.0001)$. It is important to highlight that, during the initial 6 weeks, only anti-HER2 therapy was given without chemotherapy, allowing evaluation of tumor response based on anti-HER2 therapy alone. Subgroup analysis consistently showed higher pCR rates for dual therapy when patients were stratified by hormone receptor (estrogen receptor [ER]) status, involvement of axillary lymph nodes, clinical tumor size, and suitability for BCS. ${ }^{30}$ There was no difference in the number of patients undergoing BCS or the rate of conversion to BCS across treatment arms. Interestingly, the higher pCR rates in the combination arm of approximately $50 \%$ did not translate into a correspondingly higher rate of BCS. However, a similar outcome has been noted in many other modern trials of neoadjuvant therapy. ${ }^{28}$ Several factors, including tumor characteristics prior to neoadjuvant treatment and patients' wishes, have been implicated as a major determinant of type of surgery. ${ }^{31}$ The correlation of pCR with event-free survival (EFS) and OS in the NeoALTTO trial has been recently reported..$^{32} \mathrm{After}$ a median follow-up of 3.77 years (for EFS) and 3.84 years (for OS), there was a statistically significant improvement in both EFS and OS in women who achieved pCR compared with those who did not (hazard ratio [HR] 0.38 [EFS] and 0.35 [OS], respectively). Interestingly, there was no significant difference in outcomes seen in patients who received dual blockade therapy versus either lapatinib or trastuzumab alone. The best outcome was noted in patients with HER2+, hormone receptor-negative (ER-) tumors treated with combination therapy (3-year EFS, 86\%).

The results of NeoALTTO were reiterated by the Chemotherapy, Herceptin and Lapatinib in Operable Breast Cancer (CHER-LOB) trial, a randomized phase II trial that also evaluated the combination of lapatinib and trastuzumab therapy in HER2+ tumors in the preoperative setting. ${ }^{33}$ Patients with Stage II-IIIA BC $(n=121)$ were assigned to receive 12 weeks of paclitaxel followed by four cycles of FEC given concurrently with trastuzumab ( $\operatorname{arm} \mathrm{A}$ ), lapatinib ( $\operatorname{arm} B$ ), or the combination ( $\operatorname{arm} C$ ) for 26 weeks. This treatment regimen differed from NeoALTTO in that all patients received FEC prior to surgery. Despite differences in therapy regimens, CHER-LOB also demonstrated significant improvement in pCR (breast and axilla) with dual therapy compared to trastuzumab or lapatinib alone ( $46.7 \%$ vs $25 \%$ vs $26.3 \%$, respectively; $P=0.019$ ) demonstrating an $80 \%$ increase in $\mathrm{pCR}$ rate. The rate of node negativity at surgery was $72.2 \%, 71 \%$, and $84.4 \%$ in arms A, B, and C, respectively. ${ }^{33}$

Another trial, the National Surgical Adjuvant Breast and Bowel Project B41 trial (NSABP-B41), investigated the combination of trastuzumab and lapatinib and its effect on $\mathrm{pCR}$ when given with an anthracycline-taxane-based neoadjuvant chemotherapy regimen. ${ }^{34}$ However, in contrast to CHER$\mathrm{LOB},{ }^{33}$ the anthracycline was given prior to HER2-targeted therapy. In this study, ${ }^{34} 529$ patients with HER2+ operable $\mathrm{BC}$ received neoadjuvant doxorubicin and cyclophosphamide every 3 weeks for four cycles followed by weekly paclitaxel for 12 weeks, concurrent with either weekly trastuzumab, lapatinib, or the combination. Following surgery, all patients received trastuzumab every 3 weeks for a total of 52 weeks of HER2 therapy. In contrast to CHER-LOB ${ }^{33}$ or NeoALTTO, ${ }^{30}$ while there was a trend toward benefit (numerically higher pCR rates), there was no statistically significant difference in either pCR-breast or pCR-breast + lymph nodes with 
combination anti-HER therapy compared to trastuzumab alone ( $62 \%$ vs $52.5 \%, P=0.095$, breast; and $60.2 \%$ vs $49.4 \%$, $P=0.056$, breast + lymph nodes). Subgroup analysis showed that $\mathrm{pCR}$ improvement with the addition of lapatinib to trastuzumab occurred mainly in clinically node-positive patients (47.2\% vs 67.5\%; OR 2.32). Similar rates of clinical overall response were reported in all three groups. However, fewer patients in the lapatinib group achieved a clinical CR $(69.9 \%)$ as compared to the combination group (82\%) or trastuzumab-alone group (76.8\%). ${ }^{34}$

In the Cancer and Leukemia Group B 40601 trial (CALGB-40601), a phase II, randomized trial, 305 patients with Stage II-III HER2+ BC were assigned to paclitaxel with trastuzumab alone or in combination with lapatinib for 16 weeks prior to surgery. ${ }^{35}$ The third arm, consisting of lapatinib and paclitaxel, was closed early due to negative efficacy and toxicity of this combination, based on results observed early on in the Adjuvant Lapatinib and/or Trastuzumab Treatment Optimisation (ALTTO) trial. ${ }^{36}$ Following surgery, all patients received four cycles of dose-dense doxorubicin and cyclophosphamide followed by a recommended 1-year treatment of trastuzumab. Numerically higher rates of pCR (breast) were observed in patients treated with combined trastuzumab and lapatinib (51\%) compared to trastuzumab alone (40\%), but similar to NSABP-B41, this difference was not statistically significant $(P=0.11) .{ }^{35}$

TRIO US B07, a phase II randomized trial, treated 140 women with Stage I-III HER2+ BC with either trastuzumab alone, lapatinib alone, or the combination for 21 days followed by six cycles of docetaxel and carboplatin with the assigned HER2 therapy. ${ }^{37}$ The primary endpoint of pCR (breast and axilla) was reported to be $52 \%$ in the docetaxelcarboplatin-trastuzumab-lapatinib (DCTL) arm vs 25\% for docetaxel-carboplatin-lapatinib (DCL) arm vs 47\% for docetaxel-carboplatin-trastuzumab (DCT) arm. All patients in the DCT arm completed protocol-specified therapy, whereas only $72 \%$ of patients in DCL and $73 \%$ in DCTL arms were able to adhere to therapy. Rates of pCR were higher in the ERsubgroup (67\% DCTL vs $41 \%$ DCL vs $57 \%$ DCT) than in the ER+ subgroup (40\% DCTL vs 11\% DCL vs 40\% DCT).

Another smaller study evaluating $\mathrm{pCR}$ rates from lapatinib and trastuzumab combination randomized 100 patients with Stage II-III HER2-positive BC to 2 weeks of either lapatinib alone (1,250 mg daily), trastuzumab alone, or the combination (with lapatinib reduced to $750 \mathrm{mg}$ daily), followed by 4 cycles of FEC and 12 weekly cycles of docetaxel, with the assigned HER2 therapy given throughout. The protocol required biopsy samples to be collected at baseline (prior to start of the therapy),
2 weeks of treatment with anti-HER2 agent, and then finally at surgery. The initial analysis presented at the 2011 American Society of Clinical Oncology (ASCO) annual meeting included the 49 patients in whom the paired biopsies were analyzed, and hence, the pCR rates presented may not be representative of the entire population. Although the trial was not powered to compare $\mathrm{pCR}$ rates across arms, a higher $\mathrm{pCR}$ (breast) rate was seen in the combination arm (74\%) than in the trastuzumabalone (54\%) or lapatinib-alone (45\%) arms. ${ }^{38}$

The Translational Breast Cancer Research Consortium (TBCRC) 006 trial investigated the role of the combination of trastuzumab and lapatinib (plus letrozole in ER+ patients) without chemotherapy in the neoadjuvant setting, and demonstrated a pCR (breast) rate of $27 \%$ (17/64 patients), suggesting that this regimen may be useful in carefully selected groups of patients. ${ }^{39}$ In the ER+ group, pCR rate was predictably higher at $36 \%$ as compared to $21 \%$ in the ER- subgroup. Of note, approximately $62 \%$ of patients in this study had tumor size of $>5 \mathrm{~cm}$. Ongoing biomarker analysis is currently underway from these patient samples to facilitate identification of predictive biomarkers to anti-HER2 combination therapy alone.

In a meta-analyses of 767 patients with early stage HER2+ BC enrolled in four neoadjuvant trials $\mathrm{s}^{30,33,38}$ evaluating the effect of the addition of lapatinib to trastuzumab and neoadjuvant chemotherapy, pCR (breast and lymph nodes) rate was $38.36 \%$ in the trastuzumab arm and $55.76 \%$ in the combination arm (OR 1.94; 95\% CI 1.44-2.6; $P<0.0001) .{ }^{40}$ The three trials that evaluated pCR (breast) for the combination of lapatinib and trastuzumab resulted in a superior pCR rate of $55.01 \%$ vs $40.70 \%$ in the trastuzumab-alone arm (OR 1.78; 95\% CI 1.27-2.50; $P=0.0007)$. The odds of achieving pCR were 2.06 times higher in the ER-subgroup and 1.76 times higher in the ER+ subgroup. In a similar meta-analysis of 779 patients enrolled in NSABP-B41, ${ }^{30}$ CHER-LOB,${ }^{33}$ NeoALTTO, ${ }^{34}$ and a study by Holmes et al, ${ }^{38}$ the addition of lapatinib to neoadjuvant chemotherapy resulted in a pCR (breast and axilla) rate of $56.7 \%$ as compared to $41.2 \%$ in patients who did not receive lapatinib. ${ }^{41}$

\section{Adverse events}

In the NeoALTTO trial, a higher proportion of patients in the lapatinib-alone arm experienced diarrhea, rash, neutropenia, and liver enzyme alterations than the dual HER2 therapy arm..$^{30}$ Furthermore, $18 \%-21 \%$ of patients did not complete planned treatment due to intolerable side effects in the lapatinib-containing treatment arms. Of note, the concomitant administration of lapatinib and docetaxel may 
have contributed to the higher number of diarrhea and neutropenia cases seen in the lapatinib arm. However, no major cardiac toxicities were observed in any of the treatment arms, with only one patient in each group having a left ventricular ejection fraction (LVEF) $<50 \%$ or $>10 \%$ from baseline, and one patient in the combination group developing class III congestive heart failure (CHF); the LVEF patients recovered after therapy was stopped. ${ }^{30}$

In a sub-study published after the initial results of NeoALTTO, ${ }^{30}$ Azim et $\mathrm{al}^{42}$ reported further analyses on lapatinib-specific adverse events (AEs). Due to the design of the trial, with targeted therapies administered alone during the first 6 weeks, the toxicities of lapatinib could be examined independent of chemotherapy. The majority of patients (78.8\%) who received lapatinib developed diarrhea $(28 \%$ grade $3-4), 61.8 \%$ developed skin rash (6.8\% grade $3-4)$, and $41.2 \%$ developed hepatic toxicities (36\% grade $3-4$ ). Both rash and diarrhea mainly developed during the first 2-3 weeks of lapatinib; whereas hepatic toxicities did not develop until 6-8 weeks after starting lapatinib, indicating that hepatic toxicity was likely exacerbated by the addition of docetaxel. Interestingly, the authors also observed that younger patients ( $<50$ years of age) had significantly higher rates of rash development compared to older patients ( $>50$ years of age; $74.4 \%$ vs $47.9 \% ; P<0.0001$ ). Early onset of rash (within 6 weeks of starting therapy) was associated with improved pCR in patients $>50$ years of age, but not in those $\leq 50$ years of age. ${ }^{42}$ This association between rash and clinical response has also been demonstrated in non-small-cell lung carcinoma and head and neck cancers treated with other EGFR tyrosine kinase inhibitors, and the association could potentially be used as an early marker to identify patients who would receive the maximum benefit of lapatinib-based therapy, although this needs to be validated in future prospective trials. ${ }^{43,44}$

Also, in the NSABP-B41 trial, a lower percentage of patients in the lapatinib group received the full planned course of docetaxel (including lower mean cumulative dose) due to dose reductions and discontinuations secondary to increased toxicity. ${ }^{34}$ There was no difference in the duration of HER2 therapy or the mean cumulative dose of docetaxel between the trastuzumab control and combination groups, suggesting that the addition of lapatinib did not compromise delivery of standard agents. As predicted, grade 3 and 4 AEs were more common in the two groups with lapatinib, with diarrhea being the most common AE (27\% in combination vs $20 \%$ in the lapatinib-alone group). ${ }^{34}$ Similarly, predictable toxicities of rash, hepatic enzyme elevation, and diarrhea, as well as treatment interruption/discontinuation rates, occurred at a higher frequency in patients assigned to the lapatinib arm in the CHER-LOB study. ${ }^{33}$ The high occurrence of diarrhea necessitated a protocol amendment to reduce the starting dose of lapatinib from $1,500 \mathrm{mg}$ to $1,250 \mathrm{mg}$, and $1,000 \mathrm{mg}$ to $750 \mathrm{mg}$ in the single and combination arms, respectively. Despite this result, $17 \%$ of patients in the combination arm and $30 \%$ in the lapatinib-alone arm discontinued treatment due to AEs.

In the CALGB-40601 trial, as expected, grade 3+ toxicity was higher among lapatinib-containing arms, including neutropenia (12\% docetaxel-lapatinib [DL], 7\% docetaxeltrastuzumab-lapatinib [DTL], 2\% docetaxel-trastuzumab [DT]), rash (15\% DL, 14\% DTL, 2\% DT), and diarrhea (20\% DL, 20\% DTL, 2\% DT). ${ }^{35}$ The most common grade $3-4$ toxicity in the TRIO US B07 trial was reported to be diarrhea, occurring in 45/128 (35\%) of patients, with most events occurring in the DCTL arm (28/58). ${ }^{37}$

Based on meta-analyses of NSABP-B $41,{ }^{34} \mathrm{CHER}-\mathrm{LOB},{ }^{33}$ NeoALTTO, ${ }^{30}$ and the trial by Holmes et al, ${ }^{38}$ patients receiving lapatinib-based chemotherapy were 2.4 times more likely to experience grade 3-4 AEs of diarrhea, neutropenia, dermatologic, and hepatic toxicity than patients who did not receive lapatinib. ${ }^{41}$ It is important to note that data from these meta-analyses do not include individual patient information, and hence, caution should be exercised when interpreting the results of these studies.

\section{Trastuzumab plus pertuzumab Efficacy}

Trastuzumab in combination with pertuzumab in the neoadjuvant setting has also been evaluated (Table 2). The NeoSphere trial was a four-armed, randomized phase II clinical trial that enrolled 417 patients with stage II-III HER2+ BC to evaluate the efficacy of the combination of trastuzumab plus pertuzumab. Patients were assigned to one of four treatment arms: 1) trastuzumab alone with docetaxel; 2) pertuzumab alone with docetaxel; 3) trastuzumab and pertuzumab with docetaxel; or 4) trastuzumab and pertuzumab without chemotherapy prior to breast surgery. ${ }^{45}$ Postoperatively, all groups then went on to receive three cycles of FEC and trastuzumab for a total of 1 year, with the addition of docetaxel only in the group who did not receive docetaxel in the neoadjuvant setting. Results demonstrated that patients who were given pertuzumab and trastuzumab plus docetaxel had a significantly improved pCR (45.8\%) compared with those given trastuzumab with docetaxel $(29 \%)$ or pertuzumab with docetaxel $(24 \% ; P=0.01)$. These results were considered practice-changing and drove the FDA approval of pertuzumab for neoadjuvant treatment of HER2+ BC. ${ }^{45}$ Notably, the dual 
Table 2 Summary of phase II trials using combination trastuzumab plus pertuzumab in the neoadjuvant setting for HER2 positive breast cancer

\begin{tabular}{|c|c|c|c|c|c|c|}
\hline Study/phase & Therapy & Patients enrolled & $\begin{array}{l}\text { PCR (breast } \\
\text { only) }\end{array}$ & $\begin{array}{l}\text { pCR (breast + } \\
\text { LN) }\end{array}$ & $\begin{array}{l}\text { Rate of } \\
\text { discontinuation }\end{array}$ & ORR \\
\hline NeoSphere//I5 & $\begin{array}{l}\text { A. } \mathrm{T}+\text { docetaxel } \rightarrow \\
\text { surgery } \rightarrow \mathrm{FEC} \times 3+\mathrm{T} \\
\text { B. } \mathrm{T}+\mathrm{P}+\text { docetaxel } \rightarrow \\
\text { surgery } \rightarrow \mathrm{FEC} \times 3+\mathrm{T} \\
\text { C. } \mathrm{T}+\mathrm{P} \rightarrow \text { surgery } \rightarrow \\
\text { docetaxel } \times 4+\mathrm{T} \rightarrow \\
\mathrm{FEC} \times 3+\mathrm{T} \\
\text { D. } \mathrm{P}+\text { docetaxel } \rightarrow \\
\text { surgery } \rightarrow \mathrm{FEC} \times 3+\mathrm{T}\end{array}$ & $\begin{array}{l}\text { Stage II/III, including } \\
\text { inflammatory cancer } \\
417 \text { patients } \\
\text { (A: 107, B: 107, } \\
\text { C: I07, D: } 96 \text { ) }\end{array}$ & $\begin{array}{l}\text { A. } 29 \% \\
\text { B. } 45.8 \% \\
(P=0.0 \mathrm{I}) \\
\text { C. } 16 \% \\
\text { D. } 24 \%\end{array}$ & $\begin{array}{l}\text { A. } 21.5 \% \\
\text { B. } 39.3 \% \\
\text { C. } 11.2 \% \\
\text { D. } 17.7 \%\end{array}$ & Not reported & $\begin{array}{l}\text { Prior to surgery } \\
\text { (complete or partial- } \\
\text { by physical exam) } \\
\text { Breast Only } \\
\text { A. } 79.8 \% \\
\text { B. } 88.1 \% \\
\text { C. } 67.6 \% \\
\text { D. } 71.4 \% \\
\text { Breast }+ \text { LN } \\
\text { A. } 81.4 \% \\
\text { B. } 88.0 \% \\
\text { C. } 66.3 \% \\
\text { D. } 73.9 \%\end{array}$ \\
\hline TRYPHAENA/II ${ }^{47}$ & $\begin{array}{l}\text { A. Carboplatin }+ \\
\text { docetaxel }+\mathrm{T}+\mathrm{P} \\
\text { B. } \mathrm{FEC} \rightarrow \mathrm{T}+\mathrm{P}+ \\
\text { docetaxel } \\
\text { C. } \mathrm{FEC}+\mathrm{T}+\mathrm{P} \rightarrow \\
\mathrm{T}+\mathrm{P}+\text { docetaxel }\end{array}$ & $\begin{array}{l}\text { Stage II/III, including } \\
\text { inflammatory cancer } \\
225 \text { patients } \\
\text { (A: } 77, \text { B: } 75, \text { C: } 73 \text { ) }\end{array}$ & $\begin{array}{l}\text { A. } 61.6 \% \\
\text { B. } 57.3 \% \\
\text { C. } 66.2 \%\end{array}$ & $\begin{array}{l}\text { A. } 51.9 \% \\
\text { B. } 45.3 \% \\
\text { C. } 50.7 \%\end{array}$ & Not reported & $\begin{array}{l}\text { Prior to surgery } \\
\text { (complete or partial) } \\
\text { A. } 89.6 \% \\
\text { B. } 94.7 \% \\
\text { C. } 91.8 \%\end{array}$ \\
\hline
\end{tabular}

Note: $A, B$, and $C$ denote treatment arms $A, B$, and $C$.

Abbreviations: FEC, fluorouracil, epirubicin, cyclophosphamide; L, lapatinib; LN, lymph node; ORR, objective response rate; P, Pertuzumab; PCR, pathologic complete response; T, trastuzumab; HER2, human epidermal growth factor receptor 2.

anti-HER2 therapy without chemotherapy arm resulted in a pCR rate of $16.8 \%$, which could suggest a potential role for a treatment regimen without chemotherapy in select groups of patients who may not be candidates for cytotoxic chemotherapy. As expected, overall response in breast and lymph nodes was highest in the pertuzumab, trastuzumab, and docetaxel arm $(88 \%))^{45}$

Long-term outcomes were recently reported at the 2015 ASCO annual meeting. Three-year PFS of those given combination trastuzumab plus pertuzumab with docetaxel was $90 \%$, compared to $86 \%$ in those given trastuzumab with docetaxel (HR 0.69; 95\% CI 0.34-1.40). HRs for DFS and PFS were 0.68 (95\% CI 0.36-1.26) and 0.54 (95\% CI 0.29-1.00), respectively, for those who achieved pCR compared to those who did not. All patients were given identical adjuvant therapy, and therefore, results suggest that the benefit of adding pertuzumab in the neoadjuvant setting may persist after surgery, although to a lesser degree than initially predicted. ${ }^{46}$

Trastuzumab plus Pertuzumab in Neoadjuvant HER2Positive Breast Cancer (TRYPHAENA) was another trial designed to investigate the tolerability of neoadjuvant treatment regimens combining pertuzumab with $\mathrm{H}$ and either a standard anthracycline or platinum-based chemotherapy for HER2 positive BC. ${ }^{47}$ Patients were randomized to either 1) six cycles of docetaxel, carboplatin, trastuzumab, and pertuzumab (DCTP); 2) three cycles of FEC followed by three cycles of docetaxel plus trastuzumab/pertuzumab (FEC $\rightarrow$ DTP); or 3) three cycles of FEC followed by three cycles of docetaxel, all concurrently with trastuzumab/pertuzumab (FEC-TP $\rightarrow$ DTP), resulting in a pCR (breast) rate of $61.6 \%$, $57.3 \%$, and $66.2 \%$, respectively. Following surgery, all patients received adjuvant trastuzumab to complete a total of 1 year of therapy. The pCR rate was higher in patients with ER- tumors compared with ER+ tumors across all arms (arm A, $81.1 \%$ vs $47.5 \%$; arm B, $62.5 \%$ vs $45.7 \%$; arm C, $73.5 \%$ vs $41.0 \%$, respectively). As all the treatment arms in this study were experimental, interpretation of efficacy results is limited, albeit encouraging, with high pCR rates seen in all arms. ${ }^{47}$

The Investigation of Serial Studies to Predict Your Therapeutic Response with Imaging and Molecular Analysis 2 (I-SPY 2) trial (clinical trial number NCT01042379), represents a distinct and personalized adaptive trial design, a paradigm shift in the way clinical trials have been conducted thus far. ${ }^{48}$ This trial includes a large array of treatment arms and assigns patients based on an algorithm designed to detect biomarkers that will predict which patients will benefit most from promising novel drugs. While this trial has not released final results, the researchers have been successful in "graduating" two drugs, veliparib and neratinib, from the study to accelerate drug development based on responses seen 
thus far. ${ }^{49,50}$ The addition of neratinib versus trastuzumab to neoadjuvant taxane-anthracycline was reported to have an estimated pCR rate of 55\% (range, 46\%-64\%) versus 32\% (range, 22\%-43\%) in ER-/HER2+ patients. ${ }^{49}$ Results of the I-SPY 2 trial regarding combination trastuzumab plus pertuzumab and TDM1 plus pertuzumab are awaited. ${ }^{48,51}$

\section{Adverse events}

Regarding the overall safety profile of trastuzumab and pertuzumab in combination, both the NeoSphere ${ }^{45}$ and the TRYPHAENA ${ }^{47}$ trials saw similar types and rates of toxicity. Diarrhea, alopecia, and nausea were seen in more than half of patients evaluated in TRYPHAENA. The most frequently reported grade 3-4AEs were neutropenia, febrile neutropenia, and leukopenia; however, all patients in this trial were receiving concurrent chemotherapy, which likely caused the majority of these toxicities. The incidence of serious AEs were highest in the DCTP arm (35.5\%). ${ }^{47}$ In NeoSphere, the most common serious AEs were the same as in TRYPHAENA; however, in the arm that did not receive chemotherapy, the rate of neutropenia, febrile neutropenia, and leukopenia was significantly lower (4\% vs $10 \%-17 \%$ in all other arms). ${ }^{45}$

\section{Predictors for response}

\section{Hormone receptor status}

Subanalyses of several neoadjuvant trials ${ }^{30,32-35,39,45,47}$ have been performed in an attempt to identify specific biomarkers that may predict which patients will respond best to HER2targeted therapy. For example, the correlation of ER status and pCR rate has been evaluated in multiple studies. ${ }^{30,32-35,39}$ In the TBCRC 006 trial, results were stratified based on ER status. The researchers were then able to demonstrate that ERpatients experienced increased $\mathrm{pCR}$ rates, despite the addition of letrozole to ER+ tumors. ${ }^{39}$ Increased pCR in ER- tumors was also seen in NeoALTTO ${ }^{30}$ and the NSABP-B41 trial. ${ }^{34}$ In NeoALTTO, the pCR rate was $61.3 \%$ in the ER- group vs $41.6 \%$ in the ER+ group. There was an impressive $18.9 \%$ and $24.8 \%$ difference in pCR between docetaxel plus lapatinib and docetaxel alone in the ER+ group and ER- subgroups, respectively. ${ }^{30}$ In CHER-LOB, the pCR rate was also higher in the ER- group (41.3\% vs $28.8 \%$ in the ER+ group). ${ }^{33}$

In the CALGB-40601 trial, HER2 expression was noted to vary significantly based on the presence of ERs, with only $17 \%$ of ER+ tumors exhibiting a HER2-enriched subtype, compared to $51 \%$ of ER- tumors. This result would again indicate that patients with ER-tumors would be more likely to respond to therapy. ${ }^{35,52}$ Numerically higher $\mathrm{pCR}$ rates were observed in the ER- subsets (77\% in the combination group and $55 \%$ in trastuzumab alone). ${ }^{52}$ In the NeoSphere and TRYPHAENA studies, patients with ER- disease were more likely to achieve pCR in all arms tested, a finding consistent with previous studies reported above. ${ }^{45,47}$ Specifically in NeoSphere, the dual anti-HER2 therapy and docetaxel arm resulted in a pCR rate of $63.2 \%$ in the ER- subgroup when compared to $26 \%$ in the ER+ subgroup..$^{45}$

\section{HER2 enrichment/intrinsic subtype}

Immunohistochemistry (IHC) staining on pre-treatment tumor samples from the NSABP-B41 trial indicated that targeted therapy is more efficacious in tumors with a high degree of protein expression (IHC 3+). ${ }^{34}$ This hypothesis was also supported by an analysis in the NeoALTTO trial, ${ }^{53}$ where the researchers utilized the HERmark ${ }^{\circledR}$ assay, a newer technique that provides continuous measurement of HER2 proteins to determine HER2 expression. ${ }^{53,54}$ This analysis showed that $\mathrm{pCR}$ rate increases with increasing HER2 levels, predominantly in the combination therapy arm where HER2 expression strongly correlated with pCR in both $\mathrm{ER}+($ ratio of $3.1 ; P=0.001)$ and ER- (ratio of $2.5 ; P=0.001$ ) patients. In the trastuzumab and lapatinib monotherapy arms, HER2 expression did not predict for $\mathrm{pCR}$ in the ER- patients and weakly predicted for $\mathrm{pCR}$ in the ER+ patients. This study also evaluated p95HER2, which represents the truncated form of the HER2 receptor lacking the binding site for trastuzumab. It is co-expressed in onethird of HER 2 positive tumors and is implicated in mediating trastuzumab resistance. ${ }^{12,55-57}$ A positive correlation of $\mathrm{pCR}$ with p95HER2 levels was noted only in the trastuzumab arms. Expression of p95HER2 was mainly predictive for pCR in ER+ patients treated with either trastuzumab or the combination of lapatinib plus trastuzumab, but did not predict for pCR in ERpatients. Higher HER2 and p95HER2 levels were associated with longer PFS. Interestingly, in a recent study of 445 patients with HER2+ tumors, a positive correlation between p95HER2 expression and response to trastuzumab-based neoadjuvant treatment was demonstrated. ${ }^{58}$

A hypothesis of the higher $\mathrm{pCR}$ rate observed in ER- tumors is that HER2+/ER- tumors are more likely to correspond to the true molecular HER2+ intrinsic subtype, while HER2+/ER+ tumors are often categorized as luminal $B$ intrinsic subtype. In the CALGB-40601 trial, considerable heterogeneity was noted in pre-treatment biopsy samples using RNA-based signatures, with luminal B (48\%) representing the predominant subtype in the ER+ subgroup, while the HER2-enriched subtype (51\%) was more pronounced in the ER-subgroup. ${ }^{52}$ Overall, HER2-enriched subtype tumors were significantly more likely to achieve pCR (70\%) as 
compared to luminal A (34\%) or B (36\%) subtypes. Among HER2-enriched, taxane plus trastuzumab alone produced pCR rates in excess of $70 \%$, which suggests that dual HER2 targeting or more aggressive chemotherapy may not be needed in this biologic subtype. Furthermore, in 78 patients with residual disease at surgery, there was an increase in the prevalence of luminal A and normal-like subtype in tumors compared to matched pre-treatment samples. This finding was suggested to be caused by tumor stromal alterations, intratumoral heterogeneity, cellular reprogramming, or decreased proliferation of HER2 induced by treatment. It suggests that perhaps there is cross-talk between the ER and HER pathways, and the presence of ERs may act as an "escape pathway" for tumors being hit with anti-HER therapy. ${ }^{52}$

\section{PI3K pathway}

The presence of PIK3CA mutations was analyzed as a potential predictor for response to therapy in an analysis of NeoALTTO tumor samples. ${ }^{59}$ The PIK3CA gene encodes for a constituent in the PI3K pathway and is known to be frequently mutated in BC, most notably ER+ and HER2-enriched cancers. This study demonstrated that the presence of PIK3CA mutations in breast tumors results in lower pCR after treatment with HER2-targeted therapy. The $\mathrm{pCR}$ rate decreased from $34.5 \%$ in wild-type to $21.3 \%$ in PIK3CA-mutated $(P=0.03)$ mutations. While this difference was noted across all treatment arms, it was most pronounced in the dual blockade group. ${ }^{59}$ This result is consistent with previous research, including a study published by Dave et al, ${ }^{60}$ which showed that in patients treated with neoadjuvant trastuzumab, those with low phosphatase and tensin homologue (PTEN) expression or presence of PI3K mutations were much less likely to achieve pCR compared to those with high PTEN expression or absence of PI3K mutations (18.2\% vs $66.7 \%, P=0.015)$. Similarly, in the CHER-LOB trial, patients with PIK3CA wild-type mutations who received dual anti-HER2 therapy were more likely to achieve pCR ( $48.5 \%$ vs $12.5 \% ; P=0.06$ ), although there was no difference in pCR rates between wild-type and mutated status across all treatment arms (33.3\% vs $22.7 \%$; $P=0.34) .{ }^{61}$ In contrast, an analysis of PTEN in 1,802 patients with HER2+ breast tumors treated with chemotherapy alone or chemotherapy and trastuzumab demonstrated no relationship with DFS. Nevertheless, presence of PTEN was associated with ER- and node-positive disease. ${ }^{62}$

\section{Circulating tumor cells}

Circulating tumor cells (CTCs) have been previously studied in $\mathrm{BC}$ as a potential prognostic indicator for patients undergo- ing therapy. In MBC, the detection of $\geq 5 \mathrm{CTCs} / 7.5 \mathrm{~mL}$ of blood prior to the initiation of a new treatment has been shown to increase the risk of relapse and death. ${ }^{63}$ However, two studies of CTC detection in neoadjuvant chemotherapy showed no significant relationship to $\mathrm{pCR}$, although this was not analyzed specifically in HER2+ tumors. ${ }^{64,65}$ Of the 455 patients randomized in the NeoALTTO trial, blood samples were obtained for CTC analysis in 51 (11\%) patients (12 from the trastuzumab arm, 19 from the lapatinib arm, and 20 from the combination arm). ${ }^{65}$ Patients with detected CTCs at baseline ( $\geq 1 \mathrm{CTC} / 22.5$ $\mathrm{mL}$ ) had numerically lower $\mathrm{pCR}$ rates compared to those with no CTCs, but the study was underpowered to detect statistical significance (pCR $27.3 \%$ vs $42.5 \% ; P=0.36$ ). None of the patients with detectable CTCs prior to surgery achieved a pCR. The findings of this small study need to be validated in larger studies or a meta-analysis of studies performed in a similar setting. These data suggest that identification and classification of biomarkers may help distinguish the patient populations who would benefit most from new therapeutic agents and combination treatments.

\section{Adjuvant trials}

Trastuzumab plus lapatinib

\section{Efficacy}

In the ALTTO trial, a phase III, multicenter study, the efficacy of lapatinib and trastuzumab combination was evaluated in the adjuvant setting. ${ }^{36} \mathrm{~A}$ total of 8,331 patients with operable HER2+ BC were randomized to receive either lapatinib concurrently with trastuzumab, trastuzumab followed by lapatinib, lapatinib alone, or trastuzumab alone. The randomized antiHER2 therapy was introduced either after completion of chemotherapy, concurrently with a taxane following anthracycline, or concurrently with a platinum-containing chemotherapy.

At a median follow-up time of 4.5 years, only 555 events had occurred, which was significantly lower than expected ( 850 events). ${ }^{36}$ Of note, the lapatinib-alone arm was closed early in August 2011 due to demonstrated superiority of trastuzumab over lapatinib alone, and those patients were allowed to cross over to the trastuzumab-alone arm. Approximately $40 \%$ of patients in each arm were lymph node-negative, and $45 \%$ of patients had tumors $\leq 2 \mathrm{~cm}$ in size, representing a patient population having a low risk of recurrence, and potentially translating into the lower-than-anticipated DFS event rate. Regarding the primary endpoint (reported on 6,281 patients, excluding patients enrolled in the lapatinib-alone arm), 4-year invasive DFS was $88 \%$ in those receiving combination lapatinib plus trastuzumab, compared to $86 \%$ in those receiving trastuzumab alone (HR 0.84; 97.5\% CI 0.70-1.02; $P=0.048$ ). 
DFS was noted to be $87 \%$ in the trastuzumab followed by lapatinib arm (HR 0.96; $P=0.610)$. In a pre-planned analysis, the non-inferiority of the combination of lapatinib and trastuzumab vs trastuzumab alone could not be established (HR for DFS 0.93; 95\% CI 0.76-1.13; $P=0.044 ; P \leq 0.025$ set for statistical significance). The analysis by ER status yielded similar results. Furthermore, no statistically significant differences in OS (for a total of 360 deaths at the time of analysis) were noted between the three arms (95\% for trastuzumab plus lapatinib, 95\% for trastuzumab $\rightarrow$ lapatinib, and 94\% for trastuzumab alone). ${ }^{36}$ The low rate of OS events in the follow-up period indicates that the study was underpowered, and perhaps significance could be reached with a longer follow-up period, although this appears highly unlikely. A protocol-specified updated efficacy analysis is expected in 2 years. Nevertheless, it is unlikely to match the promising results seen in the neoadjuvant setting by the NeoALTTO trial. ${ }^{30}$

The unexpected results of the ALLTO trial ${ }^{36}$ allow us to question both the utility of $\mathrm{pCR}$ as a surrogate marker for improved survival outcomes and the superiority of dual HER2 blockade over trastuzumab alone, at least in the adjuvant setting. Further analysis of the ALTTO trial may help identify certain subsets of patients, for example, those at high risk for disease recurrence, such as those patients with lymph node-positive $\mathrm{BC}$ who may preferentially benefit from dual blockade in the adjuvant setting. A large number of projects analyzing biomarkers and other translational studies are currently underway from these patient samples to facilitate a better understanding of the overall failure of improved outcome with dual HER2 blockade, to assess whether there are specific patients who may benefit from it by biomarker analysis, and to understand pathways of resistance to anti-HER2 therapy. ${ }^{36}$

Another question is whether the appropriate combination of anti-HER2 agents was tested in ALTTO. To specifically address this question, we look forward to the results of the Adjuvant Pertuzumab and Herceptin in Initial Therapy of breast cancer (APHINITY) trial, a randomized, double-blind, placebo-controlled, two-arm study evaluating adjuvant pertuzumab plus trastuzumab plus chemotherapy vs trastuzumab plus placebo plus chemotherapy. ${ }^{66}$ Until the results of such an important trial are presented, we believe that the routine use of pertuzumab in the adjuvant setting should at least be considered controversial.

\section{Adverse events}

In the ALTO trial only $60 \%-78 \%$ of patients received $>85 \%$ of the planned lapatinib dose, whereas $>90 \%$ received trastuzumab as planned. As expected, AEs such as diarrhea (75\% vs $50 \%$ vs $20 \%$, respectively), rash ( $55 \%$ vs $49 \%$ vs $20 \%$, respectively), and hepatobiliary toxicity (23\% vs $24 \%$ vs $16 \%$, respectively), including grade three or more AEs, were more frequent in the concurrent combination and sequential dual anti-HER2 treatment arms as opposed to H alone. ${ }^{36}$

In a phase II, single-arm trial, the tolerability of adjuvant lapatinib plus trastuzumab was evaluated in 95 patients with stage I-III HER2+ BC. Patients received dose-dense doxorubicin and cyclophosphamide followed by weekly docetaxel with trastuzumab and lapatinib for 12 weeks and then trastuzumab and lapatinib alone for an additional 40 weeks. In patients who developed any unacceptable AEs related to lapatinib, the drug was held temporarily, and a single-dose reduction was allowed. After a median follow-up time of 22 months, 84 patients ( $88 \%$ of evaluable patients) experienced diarrhea, with $29 \%$ of patients experiencing grade 3 diarrhea. Forty-one patients (45\%) withdrew from the study due to grade 3 or unacceptable grade 2 or less diarrhea, despite active diarrhea management, and $43 \%$ had reductions in lapatinib dose, necessitating early closure of the study. ${ }^{67}$ Three patients ( $3 \%$ ) had grade 3 rash, and four patients $(4 \%)$ had grade 2 unacceptable rash, which required a lapatinib dose reduction. Three patients (3\%) developed $\mathrm{CHF}$, and six patients (7\%) had significant asymptomatic LVEF decline during therapy, with LVEF recovery reported in three patients. No cardiac deaths were reported.

The Mayo Clinic Cancer Research Consortium 0639 (RC0639) trial, a randomized, phase II, single-arm trial, investigated the combination of lapatinib plus trastuzumab with standard chemotherapy in the adjuvant setting. ${ }^{68}$ Patients with stages I-III HER2+ BC (109 patients) received four cycles of doxorubicin and cyclophosphamide followed by 12 weeks of docetaxel, trastuzumab, and lapatinib, and then 40 weeks of trastuzumab and lapatinib. In regards to overall toxicity, grade 3-4 diarrhea was observed in 41\% and $2.7 \%$ of patients, respectively, at the initial dose of lapatinib $1,000 \mathrm{mg} /$ day. Lapatinib was then reduced to $750 \mathrm{mg} /$ day with more aggressive management of diarrhea, resulting in a decrease of grade 3 diarrhea to $31 \%$ and no additional grade 4 events. The most common hematologic AEs were leukopenia/neutropenia $(22.9 \%-27.5 \%$ grade $3 / 4)$, while liver transaminitis was limited to grade $1 / 2$ events. $^{68}$

\section{Cardiac toxicity of dual HER2 blockade}

Given the known cardiac toxicity of trastuzumab, there is reasonable concern that this toxicity would be exacerbated by the addition of another agent with a similar mechanism of action. However, results obtained from trials utilizing the combination of trastuzumab and pertuzumab actually demonstrated minimal cardiac toxicity with the combination. ${ }^{47,69}$ For 
example, in the TRYPHAENA trial, ${ }^{47} \mathrm{LVEF}$ decline of $>10 \%$ and a drop to $<50 \%$ LVEF occurred in $6.9 \%$ of patients treated with FEC-TP $\rightarrow$ DTP, $16.0 \%$ treated with FEC $\rightarrow$ DTP, and $10.5 \%$ treated with DCTP. Symptomatic left ventricular systolic dysfunction (LVSD) occurred in $4.0 \%$ of patients treated with FEC $\rightarrow$ DTP, $1.3 \%$ treated with DCTP, and interestingly, in none treated with FEC-TP $\rightarrow$ DTP. However, subsequent recovery of LVEF to $>50 \%$ occurred in all but one patient. Investigators from the TRYPHAENA trial concluded that pertuzumab does not increase the rate of cardiac dysfunction compared to trastuzumab alone with standard chemotherapy in the neoadjuvant setting. ${ }^{47,69}$

This result was similar to results seen in NeoSphere, ${ }^{45}$ where in the neoadjuvant treatment period, LVSD occurred in $2.8 \%$ of patients treated with DTP, $1.1 \%$ treated with DP, $0.9 \%$ treated with DT, and $0 \%$ treated with TP alone. The average maximum LVEF decrease was relatively low at $4 \%-5 \%$ across all treatment arms, with no significant difference between groups treated with trastuzumab alone compared to combination trastuzumab and pertuzumab. ${ }^{45}$ In the overall treatment period, LVEF declined $>10 \%$ and a drop to $<50 \%$ occurred in $1.9 \%$ of patients treated with DT vs $8.4 \%$ treated with DTP; however, LVEF subsequently recovered to $\geq 50 \%$ in all patients. Similar low rates of cardiac dysfunction were also seen when this drug combination (DTP) was given in the metastatic setting in the phase III CLEOPATRA trial, ${ }^{25}$ as well as in a meta-analysis of patients treated with combination pertuzumab/trastuzumab. ${ }^{70}$ In the NeoALTTO trial, no major cardiac toxicities were observed in any of the treatment arms, with only one patient in each group having a LVEF $<50 \%$ or $>10 \%$ from baseline, and one patient in the combination group developing class III CHF; however, LVEF recovered after therapy was stopped. ${ }^{30}$ The incidence of primary cardiac events in the ALTTO trial, defined as New York Heart Association (NYHA) class III/IV $\mathrm{CHF}$ or cardiac death, were remarkably low in all arms $(<1 \%$ of patients in each arm), despite the fact that $97 \%$ of patients had received anthracyclines. ${ }^{36}$

Likewise, no CHF episodes were reported with concurrent administration of anthracyclines and HER2-blocking agents in the CHER-LOB trial; however, the small number of patients in this study precludes any reliable conclusions. ${ }^{33}$ In the NSABP-B41 study, NYHA class III/IV CHF was reported in one patient $(1 \%)$ in the combination group and seven patients (4\%) each in the single agent lapatinib and trastuzumab groups. ${ }^{34}$ Thus far, no CHF events have been reported in the CALGB-40601 study. ${ }^{35}$ In the TRIO US B07 trial, the incidence of cardiac events, defined as $>10 \%$ decline in LVEF below baseline and all grades of LVSD, was also low (four cases in the DCL, three cases in the DCT, and three cases in the DCTL arm). ${ }^{37}$

Valachis et $\mathrm{al}^{71}$ conducted a meta-analysis to evaluate the cardiac toxicity of dual anti-HER 2 agents when compared to a single anti-HER2 agent, including four neoadjuvant trials (CHER-LOB,${ }^{33}$ NeoALTTO,${ }^{30}$ NSABP-B $41,{ }^{34}$ NeoSphere ${ }^{45}$ ). No evidence of association between dual anti-HER2 therapy and CHF ( $\geq$ grade 3 according to the National Cancer Institute-Common Toxicity Criteria (NCI-CTC), version 3; OR $0.74 ; P=0.87)$ or LVEF decline $(<50 \%$ or $\geq 10 \%$ decline from baseline; OR $1.52 ; P=0.51)$ was noted in the neoadjuvant setting.

The primary endpoint of the RC0639 trial was the cardiac safety of four cycles of doxorubicin and cyclophosphamide followed by 12 weeks of docetaxel, trastuzumab, and lapatinib, and then 40 weeks of trastuzumab and lapatinib. ${ }^{68}$ Five patients $(4.6 \%)$ in the study experienced grade 3-4 cardiac AEs during active treatment (three patients with grade 3 LVSD and two patients with grade 4 thrombosis). None of the patients developed CHF or cardiac death during active treatment; however, one patient developed confirmed CHF after discontinuing the treatment. Another patient went off study treatment due to grade 3 diarrhea, and suddenly died 2.9 months later, clinically suspected to be secondary to myocardial infarction. A statistically significant decline in baseline LVEF was noted at treatment cycle 8, treatment cycle 12, 18 months, and 5-year evaluation points. Nevertheless, the overall mean changes were less than $5 \%$ from baseline, and in most cases, were not clinically significant. ${ }^{68}$

\section{Discussion}

The primary endpoint of clinical trials evaluating the efficacy of dual HER2 blockade in the neoadjuvant setting was pCR. The use of pCR instead of PFS or OS as an endpoint carries the advantage of testing novel drugs/combinations in a relatively shorter period of time with fewer patients, thereby considerably reducing the trial cost. pCR has also been proposed to be a viable surrogate marker for survival outcomes in these trials. In a patient-based pooled analysis of over 6,300 patients enrolled in seven neoadjuvant trials, $\mathrm{pCR}$, defined as absence of invasive and in situ disease in breast and lymph node, was associated with the most superior improvement in DFS (HR 0.446) compared with patients with residual in situ disease only, no invasive residual disease in breast but involved nodes, and gross invasive residual disease, in that order. ${ }^{72}$ Achievement of pCR was associated with improved outcomes in patients with triplenegative, HER2+, and luminal B/HER2- BC, and the authors concluded that $\mathrm{pCR}$ can be used as a surrogate marker of 
DFS in these subtypes. ${ }^{72}$ These results were supported by a recent study in which approximately 12,000 patients treated in 12 neoadjuvant chemotherapy trials were evaluated, and absence of invasive disease in both breast and lymph nodes was associated with statistically significant improvement in EFS (HR 0.48) and OS (HR 0.36). This association was most pronounced in patients with triple-negative and HER2+ ERtumors. At a trial level, little association was noted between increase in frequency of pCR and EFS or OS. ${ }^{73}$ It is important to note that the variation in the definition of $\mathrm{pCR}$ across trials makes the interpretation of individual trial results more challenging, and that standardization of pCR definition may help with this issue.

In May 2012, the FDA advised that pCR (absence of invasive disease in breast and axillary lymph nodes) may be an acceptable surrogate endpoint to predict long-term efficacy in order to accelerate the approval of newer agents in patients with high-risk early stage $\mathrm{BC}$, with successive confirmatory trials demonstrating a meaningful improvement in survival outcomes. ${ }^{74,75}$ Pertuzumab subsequently gained accelerated approval in 2013 for the neoadjuvant treatment of high-risk HER2+ BC based on the results of the NeoSphere study in which pCR was utilized as a surrogate endpoint of survival outcomes. ${ }^{45}$ The National Comprehensive Cancer Network (NCCN) Clinical Practice Guidelines in Oncology (NCCN Guidelines $^{\circledR}$, version 2) recommend the use of neo-adjuvant pertuzumab in combination with trastuzumab and taxanebased chemotherapy given sequentially with anthracyclines (doxorubicin and cyclophosphamide or FEC). ${ }^{76}$

Interestingly, these guidelines also suggest that it may be reasonable to consider the use of pertuzumab in the adjuvant setting (in a similar regimen as mentioned in the paragraph above) if the patient has not received it pre-operatively for high-risk HER2+ BC (patients with $>\mathrm{T} 2$ or $>\mathrm{N} 1$ disease). However, there is no definitive data to support such an approach, and so, its routine use should be balanced against the unknown potential short- and long-term AEs and the cautionary tale of the disappointing ALTTO results. ${ }^{36}$

The much-awaited APHINITY trial results will be critical in determining the benefit of dual HER2 blockade in the adjuvant setting. The first-line combination of TDM1 plus pertuzumab and TDM1-alone arms, as tested in the phase III MARIANNE trial, showed non-inferior PFS (15.2 months, HR 0.87, 95\% CI 0.69-1.08, $P=0.14$; and 14.1 months, HR $0.91,95 \%$ CI $0.73-1.13, P=31$ ), but not superior outcomes compared to trastuzumab and taxane (13.7 months) in patients with HER2+ MBC. ${ }^{77}$ The combination of pre-operative TDM1 and pertuzumab is being actively investigated in the I-SPY 2 trial $^{48}$ and the German Adjuvant Dynamic Marker-Adjusted Personalized Therapy (ADAPT) trial optimizing risk assessment and therapy response prediction in early breast cancer (WSG AM06 trial, Fofmann et al, Trials 2013). ${ }^{78}$ Other trials are currently investigating the combination of TDM1, lapatinib, and albumin-bound paclitaxel compared with trastuzumab, lapatinib, and paclitaxel (clinical trial number NCT02073487), and neratinib with/ without trastuzumab in the neoadjuvant setting (NSABPFB7, clinical trial number NCT01008150).

All neoadjuvant trials reported thus far have shown a numerical improvement in pCR with dual HER2 blockade (trastuzumab plus lapatinib, or trastuzumab plus pertuzumab); however, the magnitude of benefit was highly variable across the trials. Possible reasons include differences in pCR definitions, tumor size, backbone of chemotherapy regimen, duration of anti-HER2 therapy, and tumor biology based on ER status. The proportion of patients with ER+ disease differed across trials, which may have influenced the pCR rate. For example, the NeoALTTO ${ }^{30}$ and NeoSphere ${ }^{45}$ trials had an equal proportion of ER+ and ER- patients, whereas over $60 \%$ of patients included in CHER-LOB, ${ }^{33}$ NSABP-B41, ${ }^{34}$ and CALGB-4060135 were ER+. Moreover, intratumoral heterogeneity has been reported in HER2+ BC, which may also influence the biologic characteristics of the disease and magnitude of response to HER2 blockade. ${ }^{79}$ Regardless of the differences in the absolute improvement in the pCR rate, these neoadjuvant studies have successfully proven the utility of dual HER2 blockade strategy in the treatment landscape of HER2+ BC. A recent literaturebased meta-analysis demonstrated a statistically significant improvement in pCR rate with dual HER2 inhibition in the range of $16 \%-19 \%$, irrespective of the chemotherapy backbone as compared to single HER2 inhibition. ${ }^{80}$ Consistent with individual studies, pCR was noted to be significantly higher in the ER- subgroup, regardless of the HER2 inhibition and type of chemotherapy. ${ }^{30,32-35,39,45,47}$

\section{Conclusion}

While dual HER2 blockade appears to be superior to single HER2 inhibition in the neoadjuvant setting, it will be critical to follow the long-term survival data of these trials. This benefit of combined HER2 blockade will need to be balanced by the increased toxicity and cost of therapy. Development of predictive biomarkers may be valuable to help identify patients who may benefit from dual HER2 therapy vs trastuzumab alone, allowing for a truly individualized treatment approach. 


\section{Disclosure}

The authors report no conflicts of interest in this work.

\section{References}

1. Ross JS, Fletcher JA. The HER-2/neu oncogene: prognostic factor, predictive factor and target for therapy. Semin Cancer Biol. 1999;9(2):125-138.

2. Ross JS, Slodkowska EA, Symmans WF, Pusztai L, Ravdin PM, Hortobagyi GN. The HER-2 receptor and breast cancer: ten years of targeted anti-HER-2 therapy and personalized medicine. Oncologist. 2009;14(4):320-368.

3. Browne BC, O'Brien N, Duffy MJ, Crown J, O'Donovan N. HER-2 signaling and inhibition in breast cancer. Curr Cancer Drug Targets. 2009;9(3):419-438.

4. Cho HS, Mason K, Ramyar KX, et al. Structure of the extracellular region of HER2 alone and in complex with the Herceptin Fab. Nature. 2003;421(6924):756-760.

5. Hudis CA. Trastuzumab - mechanism of action and use in clinical practice. $N$ Engl J Med. 2007;357(1):39-51.

6. Junttila TT, Akita RW, Parsons K, et al. Ligand-independent HER2/ HER3/PI3K complex is disrupted by trastuzumab and is effectively inhibited by the PI3K inhibitor GDC-0941. Cancer Cell. 2009;15(5): 429-440.

7. Slamon D, Eiermann W, Robert N, et al; Breast Cancer International Research Group. Adjuvant trastuzumab in HER2-positive breast cancer. N Engl J Med. 2011;365(14):1273-1283.

8. Slamon DJ, Leyland-Jones B, Shak S, et al. Use of chemotherapy plus a monoclonal antibody against HER 2 for metastatic breast cancer that overexpresses HER2. N Engl J Med. 2001;344(11):783-792.

9. Perez EA, Romond EH, Suman VJ, et al. Trastuzumab plus adjuvant chemotherapy for human epidermal growth factor receptor 2-positive breast cancer: planned joint analysis of overall survival from NSABP B-31 and NCCTG N9831. J Clin Oncol. 2014;32(33):3744-3752.

10. Goldhirsch A, Gelber RD, Piccart-Gebhart MJ, et al; Herceptin Adjuvant (HERA) Trial Study Team. 2 years versus 1 year of adjuvant trastuzumab for HER2-positive breast cancer (HERA): an open-label, randomised controlled trial. Lancet. 2013;382(9897):1021-1028.

11. Thery JC, Spano JP, Azria D, Raymond E, Penault Llorca F. Resistance to human epidermal growth factor receptor type 2-targeted therapies. Eur J Cancer. 2014;50(5):892-901.

12. Scaltriti M, Rojo F, Ocaña A, et al. Expression of p95HER2, a truncated form of the HER2 receptor, and response to anti-HER2 therapies in breast cancer. J Natl Cancer Inst. 2007;99(8):628-638.

13. Garrett JT, Sutton CR, Kuba MG, Cook RS, Arteaga CL. Dual blockade of HER2 in HER2-overexpressing tumor cells does not completely eliminate HER3 function. Clin Cancer Res. 2013;19(3):610-619.

14. Burstein HJ, Storniolo AM, Franco S, et al. A phase II study of lapatinib monotherapy in chemotherapy-refractory HER2-positive and HER2-negative advanced or metastatic breast cancer. Ann Oncol. 2008;19(6):1068-1074.

15. Barok M, Joensuu H, Isola J. Trastuzumab emtansine: mechanisms of action and drug resistance. Breast Cancer Res. 2014;16(2):209.

16. Verma S, Miles D, Gianni L, et al; EMILIA Study Group. Trastuzumab emtansine for HER2-positive advanced breast cancer. $N$ Engl $J$ Med. 2012;367(19):1783-1791.

17. Burstein HJ, Sun Y, Dirix LY, et al. Neratinib, an irreversible ErbB receptor tyrosine kinase inhibitor, in patients with advanced ErbB2positive breast cancer. J Clin Oncol. 2010;28(8):1301-1307.

18. Nahta R, Esteva FJ. Trastuzumab: triumphs and tribulations. Oncogene. 2007;26(25):3637-3643

19. Xia W, Gerard CM, Liu L, Baudson NM, Ory TL, Spector NL. Combining lapatinib (GW572016), a small molecule inhibitor of ErbB1 and ErbB2 tyrosine kinases, with therapeutic anti-ErbB2 antibodies enhances apoptosis of ErbB2-overexpressing breast cancer cells Oncogene. 2005;24(41):6213-6221.
20. Konecny GE, Pegram MD, Venkatesan N, et al. Activity of the dual kinase inhibitor lapatinib (GW572016) against HER-2-overexpressing and trastuzumab-treated breast cancer cells. Cancer Res. 2006; 66(3):1630-1639.

21. Scaltriti M, Verma C, Guzman M, et al. Lapatinib, a HER2 tyrosine kinase inhibitor, induces stabilization and accumulation of HER2 and potentiates trastuzumab-dependent cell cytotoxicity. Oncogene. 2009;28(6):803-814.

22. Arpino G, Gutierrez C, Weiss H, et al. Treatment of human epidermal growth factor receptor 2-overexpressing breast cancer xenografts with multiagent HER-targeted therapy. J Natl Cancer Inst. 2007;99(9): 694-705.

23. Rimawi MF, Wiechmann LS, Wang YC, et al. Reduced dose and intermittent treatment with lapatinib and trastuzumab for potent blockade of the HER pathway in HER2/neu-overexpressing breast tumor xenografts. Clin Cancer Res. 2011;17(6):1351-1361.

24. Blackwell KL, Burstein HJ, Storniolo AM, et al. Randomized study of Lapatinib alone or in combination with trastuzumab in women with ErbB2-positive, trastuzumab-refractory metastatic breast cancer. J Clin Oncol. 2010;28(7):1124-1130.

25. Swain SM, Baselga J, Kim SB, et al; CLEOPATRA Study Group. Pertuzumab, trastuzumab, and docetaxel in HER2-positive metastatic breast cancer. N Engl J Med. 2015;372(8):724-734.

26. Mieog JS, van der Hage JA, van de Velde CJ. Preoperative chemotherapy for women with operable breast cancer [review]. Cochrane Database Syst Rev. 2007;2:CD005002.

27. Mauri D, Pavlidis N, Ioannidis JP. Neoadjuvant versus adjuvant systemic treatment in breast cancer: a meta-analysis. J Natl Cancer Inst. 2005;97(3):188-194.

28. Untch M, Fasching PA, Konecny GE, et al. Pathologic complete response after neoadjuvant chemotherapy plus trastuzumab predicts favorable survival in human epidermal growth factor receptor 2-overexpressing breast cancer: results from the TECHNO trial of the AGO and GBG study groups. J Clin Oncol. 2011;29(25):3351-3357.

29. Gianni L, Eiermann W, Semiglazov V, et al. Neoadjuvant chemotherapy with trastuzumab followed by adjuvant trastuzumab versus neoadjuvant chemotherapy alone, in patients with HER2-positive locally advanced breast cancer (the NOAH trial): a randomised controlled superiority trial with a parallel HER2-negative cohort. Lancet. 2010;375(9712):377-384.

30. Baselga J, Bradbury I, Eidtmann H, et al; NeoALTTO Study Team. Lapatinib with trastuzumab for HER2-positive early breast cancer (NeoALTTO): a randomised, open-label, multicentre, phase 3 trial Lancet. 2012;379(9816):633-640.

31. Criscitiello C, Azim HA Jr, Agbor-Tarh D, et al. Factors associated with surgical management following neoadjuvant therapy in patients with primary HER2-positive breast cancer: results from the NeoALTTO phase III trial. Ann Oncol. 2013;24(8):1980-1985.

32. de Azambuja E, Holmes AP, Piccart-Gebhart M, et al. Lapatinib with trastuzumab for HER2-positive early breast cancer (NeoALTTO) survival outcomes of a randomised, open-label, multicentre, phase 3 trial and their association with pathological complete response. Lancet Oncol. 2014;15(10):1137-1146.

33. Guarneri V, Frassoldati A, Bottini A, et al. Preoperative chemotherapy plus trastuzumab, lapatinib, or both in human epidermal growth factor receptor 2-positive operable breast cancer: results of the randomized phase II CHER-LOB study. J Clin Oncol. 2012;30(16):1989-1995.

34. Robidoux A, Tang G, Rastogi P, et al. Lapatinib as a component of neoadjuvant therapy for HER2-positive operable breast cancer (NSABP protocol B-41): an open-label, randomised phase 3 trial. Lancet Oncol. 2013;14(12):1183-1192.

35. Carey LA, Berry DA, Ollila D, et al. Clinical and translational results of CALGB 40601: a neoadjuvant phase III trial of weekly paclitaxel and trastuzumab with or without lapatinib for HER2-positive breast cancer. J Clin Oncol. 2013;31(15 Suppl):500.

36. Piccart-Gebhart MJ, Holmes AP, Baselga J, et al. First results from the phase III ALTTO trial (BIG 2-06; NCCTG [Alliance] N063D) comparing one year of anti-HER2 therapy with lapatinib alone (L), trastuzumab alone $(\mathrm{T})$, their sequence $(\mathrm{T} \rightarrow \mathrm{L})$, or their combination $(\mathrm{T}+\mathrm{L})$ in the adjuvant treatment of HER2-positive early breast cancer (EBC). J Clin Oncol. 2014;32(15 Suppl):Abstract LBA4. 
37. Hurvitz S, Miller JM, Dichmann R, et al. Final analysis of a phase II 3 -arm, randomized trial of neoadjuvant trastuzumab or lapatinib or the combination of trastuzumab and lapatinib, followed by six cycles of docetaxel and carboplatin with trastuzumab and/or lapatinib in patients with HER2 + breast cancer (TRIO-US B07). Cancer Res. 2013;73 (24 Suppl):Abstract S1-S02.

38. Holmes FA, Nagarwala YM, Espina VA, et al. Correlation of molecular effects and pathologic complete response to preoperative lapatinib and trastuzumab, separately and combined prior to neoadjuvant breast cancer chemotherapy. J Clin Oncol. 2011;29(15 Suppl):506.

39. Rimawi MF, Mayer IA, Forero A, et al. Multicenter phase II study of neoadjuvant lapatinib and trastuzumab with hormonal therapy and without chemotherapy in patients with human epidermal growth factor receptor 2-overexpressing breast cancer: TBCRC 006. J Clin Oncol. 2013;31(14):1726-1731.

40. Hicks M, Macrae ER, Abdel-Rasoul M, et al. Neoadjuvant dual HER2targeted therapy with lapatinib and trastuzumab improves pathologic complete response in patients with early stage HER2-positive breast cancer: a meta-analysis of randomized prospective clinical trials. Oncologist. 2015;20(4):337-343.

41. Sun J, Chen C, Yao X, Sun S. Lapatinib combined with neoadjuvant paclitaxel-trastuzumab-based chemotherapy in patients with human epidermal growth factor receptor 2-positive breast cancer: A meta-analysis of randomized controlled trials. Oncol Lett. 2015;9(3):1351-1358.

42. Azim HA Jr, Agbor-Tarh D, Bradbury I, et al. Pattern of rash, diarrhea, and hepatic toxicities secondary to lapatinib and their association with age and response to neoadjuvant therapy: analysis from the NeoALTTO trial. J Clin Oncol. 2013;31(36):4504-4511.

43. Liu H, Wu Y, Lv T, et al. Skin rash could predict the response to EGFR tyrosine kinase inhibitor and the prognosis for patients with non-small cell lung cancer: a systematic review and meta-analysis. Kuwano M, ed. PLoS ONE. 2013;8(1):e55128.

44. Burtness B, Goldwasser MA, Flood W, et al. Phase III randomized trial of cisplatin plus placebo compared with cisplatin plus cetuximab in metastatic/recurrent head and neck cancer: an Eastern Cooperative Oncology Group study. J Clin Oncol. 2005;23(34):8646-54. Erratum In: J Clin Oncol. 2006;24(4):724.

45. Gianni L, Pienkowski T, Im YH, et al. Efficacy and safety of neoadjuvant pertuzumab and trastuzumab in women with locally advanced, inflammatory, or early HER2-positive breast cancer (NeoSphere): a randomised multicentre, open-label, phase 2 trial. Lancet Oncol. 2012;13(1):25-32.

46. Gianni L, Pienkowski T, Im YH, et al. Five-year analysis of the phase II NeoSphere trial evaluating four cycles of neoadjuvant docetaxel (D) and/or trastuzumab (T) and/or pertuzumab (P). J Clin Oncol. 2015;33 (15 Suppl):505.

47. Schneeweiss A, Chia S, Hickish T, et al. Pertuzumab plus trastuzumab in combination with standard neoadjuvant anthracycline-containing and anthracycline-free chemotherapy regimens in patients with HER2positive early breast cancer: a randomized phase II cardiac safety study (TRYPHAENA). Ann Oncol. 2013;24(9):2278-2284.

48. ClinicalTrials.gov [database on the Internet]. I-SPY 2 TRIAL: neoadjuvant and personalized adaptive novel agents to treat breast cancer. Bethesda, MD: US National Institutes of Health; 2009. Available from: http://www. ClinicalTrials.gov/show/NCT01042379. Accessed May 1, 2015.

49. Park JW, Liu MC, Yee D, et al. Neratinib plus standard neoadjuvant therapy for high-risk breast cancer: efficacy results from the I-SPY 2 trial. Cancer Res. 2014;74(19 Suppl):Abstract CT227.

50. Rugo HS, Olopade O, DeMichele A, et al. Veliparib/carboplatin plus standard neoadjuvant therapy for high-risk breast cancer: first efficacy results from the I-SPY 2 trial. Cancer Res. 2013;73(24 Suppl):Abstract $\mathrm{S} 5-\mathrm{S} 02$.

51. Joensuu H. Dual targeting of HER2 with lapatinib and trastuzumab. Lancet Oncol. 2014;15(10):1050-1052.

52. Carey LA, Barry WT, Pitcher B, et al; Alliance for Clinical Trials in Oncology. Gene expression signatures in pre- and post-therapy (Rx) specimens from CALGB 40601 (Alliance), a neoadjuvant phase III trial of weekly paclitaxel and trastuzumab with or without lapatinib for HER2positive breast cancer (BrCa). J Clin Oncol. 2014;32(15 Suppl):506.
53. Scaltriti M, Nuciforo P, Bradbury I, et al. High HER2 expression correlates with response to the combination of lapatinib and trastuzumab. Clin Cancer Res. 2015;21(3):569-576.

54. Shi Y, Huang W, Tan Y, et al. A novel proximity assay for the detection of proteins and protein complexes: quantitation of HER1 and HER2 total protein expression and homodimerization in formalin-fixed, paraffin-embedded cell lines and breast cancer tissue. Diagn Mol Pathol. 2009;18(1):11-21.

55. Arribas J, Baselga J, Pedersen K, Parra-Palau JL. p95HER2 and breast cancer. Cancer Res. 2011;71(5):1515-1519.

56. Pedersen K, Angelini PD, Laos S, et al. A naturally occurring HER2 carboxy-terminal fragment promotes mammary tumor growth and metastasis. Mol Cell Biol. 2009;29(12):3319-3331.

57. Parra-Palau JL, Pedersen K, Peg V, et al. A major role of p95/611-CTF, a carboxy-terminal fragment of HER2, in the down-modulation of the estrogen receptor in HER2-positive breast cancers. Cancer Res. 2010;70(21):8537-8546.

58. Loibl S, Bruey J, Von Minckwitz G, et al. Validation of $\mathrm{p} 95$ as a predictive marker for trastuzumab-based therapy in primary HER2-positive breast cancer: a translational investigation from the neoadjuvant GeparQuattro study. J Clin Oncol. 2011;29(15 Suppl):530.

59. Majewski IJ, Nuciforo P, Mittempergher L, et al. PIK3CA mutations are associated with decreased benefit to neoadjuvant human epidermal growth factor receptor 2-targeted therapies in breast cancer. $J$ Clin Oncol. 2015;33(12):1334-1339.

60. Dave B, Migliaccio I, Gutierrez MC, et al. Loss of phosphatase and tensin homolog or phosphoinositol-3 kinase activation and response to trastuzumab or lapatinib in human epidermal growth factor receptor 2-overexpressing locally advanced breast cancers. J Clin Oncol. 2011;29(2):166-173.

61. Guarneri V, Dieci MV, Carbognin L, et al. Activity of neoadjuvant lapatinib (L) plus trastuzumab (T) for early breast cancer (EBC) according to PIK3CA mutations: pathological complete response (pCR) rate in the CherLOB study and pooled analysis of randomized trials. Ann Oncol. 2014;25(Suppl 4):iv85.

62. Perez EA, Dueck AC, McCullough AE, et al. Impact of PTEN protein expression on benefit from adjuvant trastuzumab in early-stage human epidermal growth factor receptor 2-positive breast cancer in the North Central Cancer Treatment Group N9831 trial. J Clin Oncol. 2013;31(17):2115-2122.

63. Cristofanilli M, Budd GT, Ellis MJ, et al. Circulating tumor cells, disease progression, and survival in metastatic breast cancer. $N$ Engl J Med. 2004;351(8):781-791.

64. Riethdorf S, Müller V, Zhang L, et al. Detection and HER2 expression of circulating tumor cells: prospective monitoring in breast cancer patients treated in the neoadjuvant GeparQuattro trial. Clin Cancer Res. 2010;16(9):2634-2645.

65. Azim HA Jr, Rothé F, Aura CM, et al. Circulating tumor cells and response to neoadjuvant paclitaxel and HER2-targeted therapy: a sub-study from the NeoALTTO phase III trial. Breast. 2013;22(6): 1060-1065.

66. ClinicalTrials.gov [database on the Internet]. A study of pertuzumab in addition to chemotherapy and herceptin (trastuzumab) as adjuvant therapy in patients with HER2-positive primary breast cancer. Bethesda, MD: US National Institutes of Health; 2011. Available from: http://www. ClinicalTrials.gov/show/NCT01358877. Accessed May 1, 2015.

67. Dang C, Lin N, Moy B, et al. Dose-dense doxorubicin and cyclophosphamide followed by weekly paclitaxel with trastuzumab and lapatinib in HER2/neu-overexpressed/amplified breast cancer is not feasible because of excessive diarrhea. J Clin Oncol. 2010;28(18):2982-2988.

68. Moreno-Aspitia A, Dueck AC, Ghanem-Cañete I, et al. RC0639: phase II study of paclitaxel, trastuzumab, and lapatinib as adjuvant therapy for early stage HER2-positive breast cancer. Breast Cancer Res Treat. 2013;138(2):427-435.

69. Perjeta.com [database on the Internet]. Neoadjuvant breast cancer treatment: Tryphaena trial overview. Basel, Switzerland: Hoffman-La Roche Ltd; 2015. Available from: http://www.perjeta.com/hcp/neoadjuvant/ tryphaena;jsessionid=C9DC7FA332D52237F59F3D2D255C52B2. gxe501b-m2. Accessed May 1, 2015. 
70. Lenihan D, Suter T, Brammer M, Neate C, Ross G, Baselga J. Pooled analysis of cardiac safety in patients with cancer treated with pertuzumab. Ann Oncol. 2012;23(3):791-800.

71. Valachis A, Nearchou A, Polyzos NP, Lind P. Cardiac toxicity in breast cancer patients treated with dual HER2 blockade. Int J Cancer. 2013; 133(9):2245-2252.

72. von Minckwitz G, Untch M, Blohmer JU, et al. Definition and impact of pathologic complete response on prognosis after neoadjuvant chemotherapy in various intrinsic breast cancer subtypes. J Clin Oncol. 2012;30(15):1796-1804.

73. Cortazar P, Zhang L, Untch M, et al. Pathological complete response and long-term clinical benefit in breast cancer: the CTNeoBC pooled analysis. Lancet. 2014;384(9938):164-172.

74. US Food and Drug Administration [webpage on the Internet]. Guidance for Industry: Pathological Complete Response in Neoadjuvant Treatment of High-Risk Early-Stage Breast Cancer: Use as an Endpoint to Support Accelerated Approval 2014. White Oak, MD: US Food and Drug Administration; 2014. Available from: http://www.fda.gov/downloads/drugs/ guidancecomplianceregulatoryinformation/guidances/ucm305501.pdf. Accessed May 1, 2015.

75. Prowell TM, Pazdur R. Pathological complete response and accelerated drug approval in early breast cancer. $N$ Engl J Med. 2012;366(26):2438-2441.
76. Gradishar WJ, Anderson BO, Balassanian R, et al. Breast cancer version 2 . 2015. J Natl Compr Canc Netw. 2015;13(4):448-475.

77. Ellis PA, Barrios CH, Eiermann W, et al. Phase III, randomized study of trastuzumab emtansine (T-DM1) $\{+/-\}$ pertuzumab (P) vs trastuzumab + taxane (HT) for first-line treatment of HER2-positive MBC: primary results from the MARIANNE study. J Clin Oncol. 2015;33 (15 Suppl):507.

78. Hofmann D, Nitz U, Gluz O, et al. WSG ADAPT - adjuvant dynamic marker-adjusted personalized therapy trial optimizing risk assessment and therapy response prediction in early breast cancer: study protocol for a prospective, multi-center, controlled, non-blinded, randomized, investigator initiated phase II/III trial. Trials. 2013;14:261.

79. Cancer Genome Atlas Network. Comprehensive molecular portraits of human breast tumours. Nature. 2012;490(7418):61-70.

80. Bria E, Carbognin L, Furlanetto J, et al. Impact of neoadjuvant single or dual HER2 inhibition and chemotherapy backbone upon pathological complete response in operable and locally advanced breast cancer: Sensitivity analysis of randomized trials. Cancer Treat Rev. 2014;40(7):847-856.
Breast Cancer: Targets and Therapy

\section{Publish your work in this journal}

Breast Cancer: Targets and Therapy is an international, peerreviewed open access journal focusing on breast cancer research, identification of therapeutic targets and the optimal use of preventative and integrated treatment interventions to achieve improved outcomes, enhanced survival and quality of life for the cancer patient.

\section{Dovepress}

View the full aims and scopes of this journal here. The manuscript management system is completely online and includes a very quick and fair peer-review system, which is all easy to use. Visit http:// www.dovepress.com/testimonials.php to read real quotes from published authors.

Submit your manuscript here: http://www.dovepress.com/breast-cancer---targets-and-therapy-journal 